\title{
Managing chest drains on medical wards
}

The introduction of this article by $\mathrm{F}$ R Millar and T Hillman (BMJ 2018;363:k4639, doi:10.1136/bmj.k4639) states: "British Thoracic Society (BTS) guidelines in the UK necessitate the use of real-time thoracic ultrasonography when inserting a chest drain." In fact, the guidelines refer to chest drain insertion for pleural fluid drainage only, in which case ultrasonography is strongly recommended. The guidelines do not, however, apply to pneumothorax drainage without fluid. Here, the evidence is mixed, and delaying placement of a chest drain for ultrasonography could be riskier for the patient. 